\title{
The Single HabitatHabitat Module Concept a Streamlined Way to Explore
}

\author{
Joe Chambliss ${ }^{1}$ \\ NASA Johnson Space Center, Houston, TX, 77058
}

\begin{abstract}
Many concepts have been proposed for exploring space. In early 2010 presidential direction called for reconsidering the approach to address changes in exploration destinations, use of new technologies and development of new capabilities to support exploration of space. Considering the proposed new technologies and capabilities that NASA was directed to pursue, the Single Habitathabitat module (SHMSHM) concept for a more streamlined approach to the infrastructure and conduct of exploration missions was developed. The SHM concept combines many of the new promising technologies with a central concept of mission architectures that uses a single habitat module for all phases of an exploration mission. Integrating mission elements near Earth and fully fueling them prior to departure of the vicinity of Earth provides the capability of using the single habitat both in transit to an exploration destination and while exploring the destination. The concept employs the capability to return the habitat and interplanetary propulsion system to Earth vicinity so that those elements can be reused on subsequent exploration missions. This paper describes the SHM concept, and the advantages it provides to accomplish exploration objectives.
\end{abstract}

\section{Nomenclature}

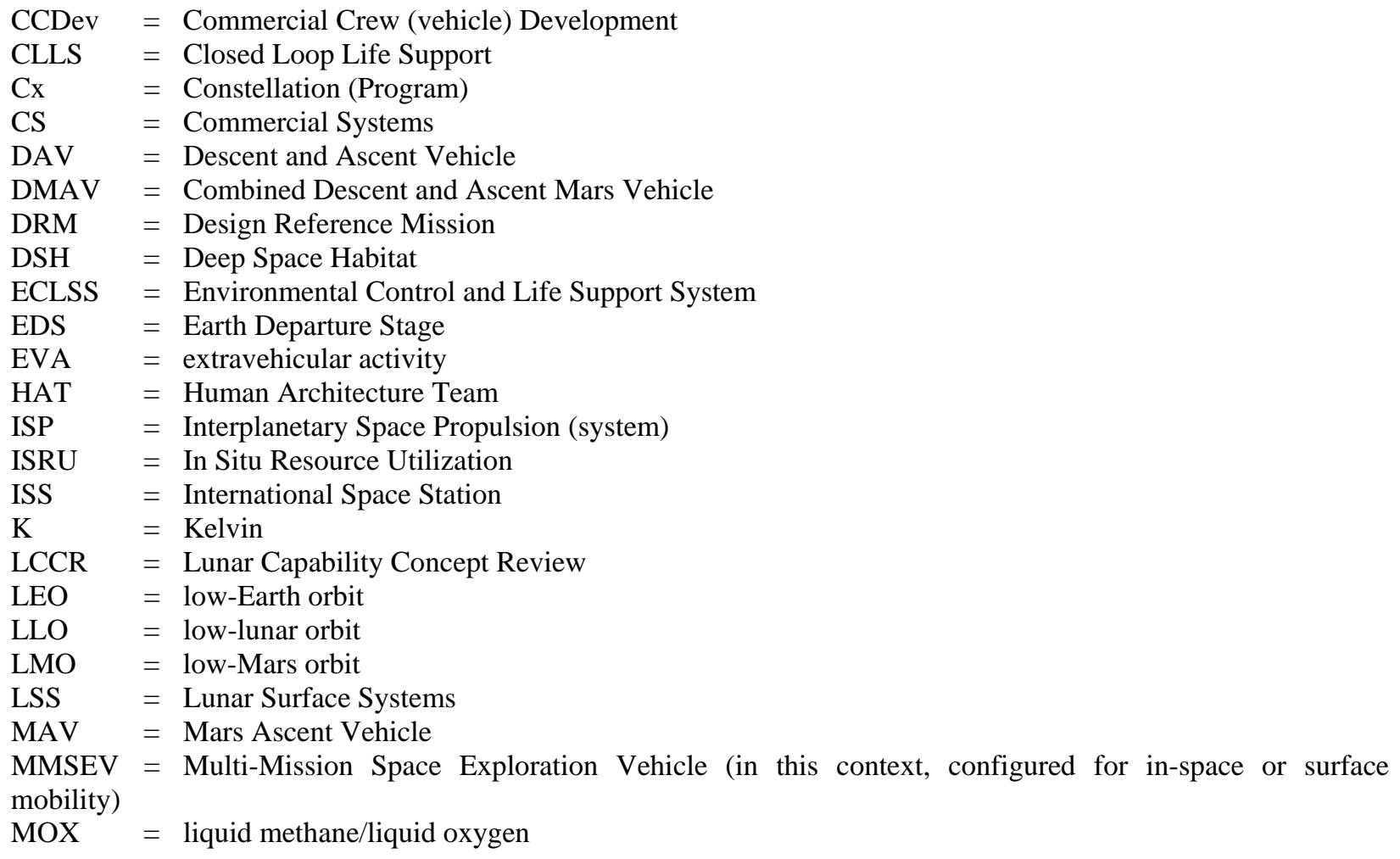

${ }^{1}$ Deputy Division System Manager for Exploration, Crew and Thermal Systems Division, 2101 NASA Parkway, Houston Texas 77062 EC1 and AIAA Associate Fellow 


$\begin{array}{ll}\text { NEA } & =\text { near-Earth asteroid } \\ \text { NTR } & =\text { Nuclear Thermal Rocket } \\ \text { OAB } & =\text { Orbiting Assembly Base } \\ \text { OAS } & =\text { Orbiting Assembly Station } \\ \text { RCS } & =\text { Reaction Control System } \\ \text { SHM } & =\text { Single Habitat module } \\ \text { SHM } & =\text { single habitat module (concept for human exploration) } \\ \text { SEV } & =\text { surface exploration vehicle } \\ \text { TCS } & =\text { Thermal Control System } \\ \text { TEI } & =\text { Trans Earth Injection (propulsion) } \\ \text { TMI } & =\text { Trans Mars Injection (propulsion) }\end{array}$

\section{Introduction}

Then humanity goes to Mars and other exploration destinations, the approach employed will affect the success of the endeavor. Combining the best ideas for the technology with an efficient approach is most likely to result in mission success.

Deep space missions require that the crew be supported in transit and at exploration destinations for long durations. The single habitat module (SHM) concept (Fig. 1) recognizes that crew support requirements for transit and while at a destination are roughly the same and thus could be addressed with a single module. Assuming the heritage of recent decades of human space operations in low-Earth orbit (LEO), the SHM concept starts with assembly of the exploration vehicle at a location near Earth using the capabilities humanity has developed and demonstrated in creating and operating the International Space Station (ISS). The SHM vehicle will include habitation to address crew support requirements and an efficient Interplanetary Space Propulsion (ISP) system to address propelling the vehicle to and from an exploration destination. Assembling and fueling the integrated vehicle near Earth provides the capability to start exploration missions fully fueled to meet the requirements of the mission. Being fully fueled at the start of each mission makes it possible to leave the vicinity of Earth with enough fuel to return a habitat from an exploration destination. Employing only one habitat can dramatically simplify mission conduct and make it possible to reuse mission assets.

The end of a SHM exploration mission results in the ISP system and habitat being returned to the near-Earth staging site. Those core elements are to be refurbished and refueled in space, then reused for subsequent exploration missions.

Such an approach to space exploration would focus development on the fewest possible number of exploration elements (the habitat and ISP) and enable reuse of those elements to provide a human exploration infrastructure that can address many exploration goals. A campaign of exploration missions using the SHM approach should be more quickly achievable and much more affordable (versus independent missions) since fewer elements are required.

This paper provides a description of the SHM concept, including ideas on how the concept could be implemented using a combination of new technologies and past exploration program concepts. Recent interactions with trajectory experts have led to better understanding of the implications of using the advanced technologies for propulsion. The resulting mission is described including changes that address the trajectory of a nuclear powered electric propulsion system and considering safety constraints for the use of nuclear powered vehicles near Earth. The description concludes with an estimate of the mass of the habitat that would be required and a plan for how using that habitat mass can lead to calculation of the mass of a habitat propulsion system to get the habitat to and from a destination orbit. The mass of the ISP can be calculated by using that habitat plus propulsion system mass. That set of calculations provides total SHM mass needed for an exploration mission and that can then be compared to other exploration concepts.

A qualitative comparison of the SHM with recent concepts for conducting exploration missions is provided to better understand the concept. A qualitative assessment of the benefits of the SHM approach and a recommendation of forward work needed to establish the benefits of the SHM approach are discussed. 


\section{Single Habitat Module Concept for Transit and Surface Operations}

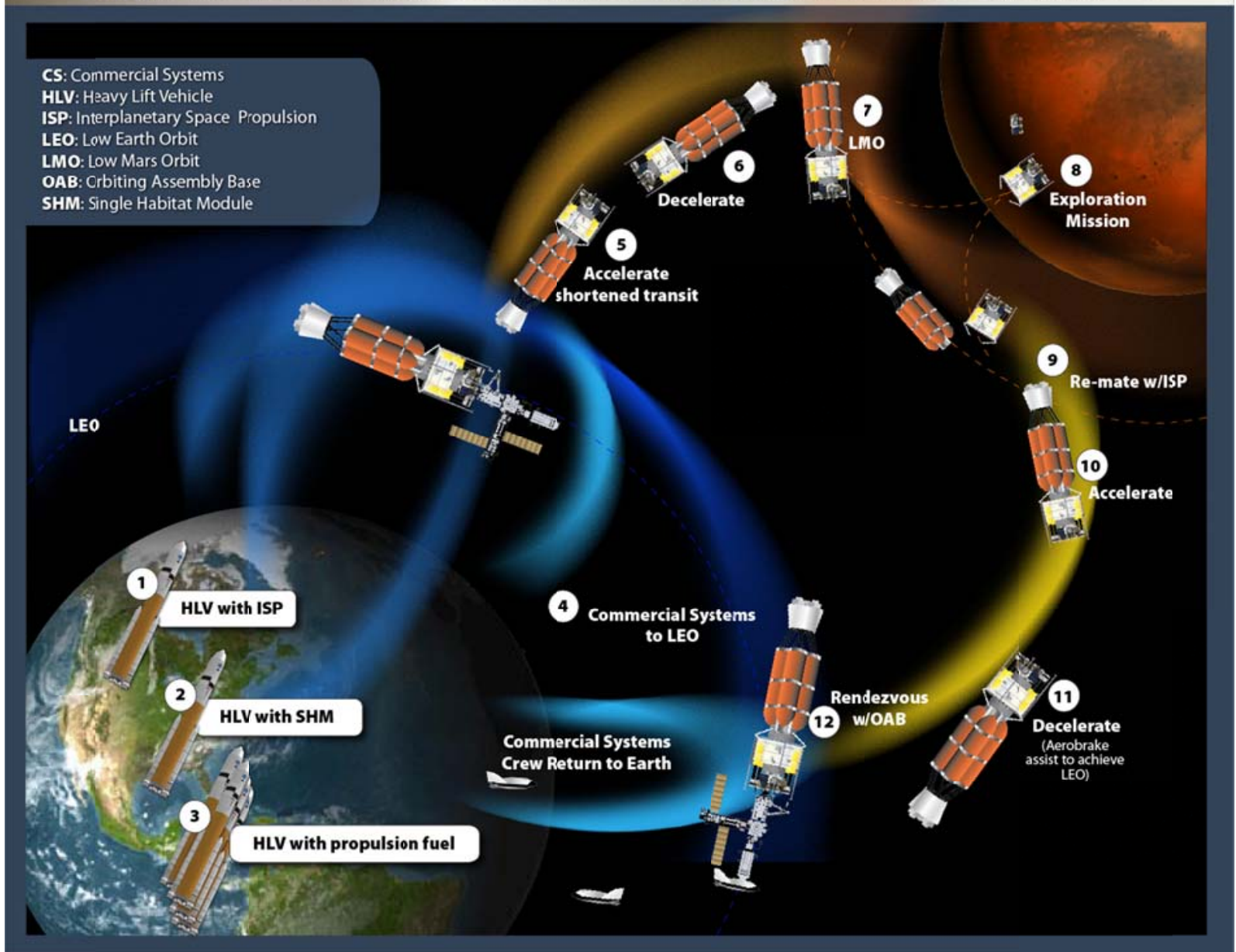

Figure 1. The SHM concept for a Mars surface mission (the design driving mission).

\section{A. Single Habitat Module Concept Mission Operations}

As illustrated in Fig. 1 for a Mars mission, each SHM mission would implement the following mission scenario:

1 thru 3 - Assemble and fuel the mission elements near Earth

Check out each element at the in-space assembly station,

4 - Deliver the exploration crew to man the vehicle via Orion or Commercial Crew Development (CCDev)

5 and 6 - Transit to the deep space destination via the ISP system

Accelerate roughly $1 / 2$ the way to destination then decelerate the rest of the transit to achieve low orbit at destination (potentially use aerocapture assist to decelerate at Mars to aid in achieving low orbit)

7 - Separate the habitat with its propulsion system from the ISP system

8 - Use the habitat propulsion system to descend and land at the exploration site on the destination surface

Conduct surface operations using the SHM as the base of operations (this is what it is all about)

9 - Ascend to low destination orbit via the habitat propulsion system and rendezvous with the ISP system

Discard the habitat propulsion system after re-mating with the ISP system to reduce return mass

(possibly discard only the prop tanks to retain the engines for reuse)

10 and 11 - Use the ISP system to return to Earth vicinity orbit

Accelerate roughly $1 / 2$ the way to Earth then decelerate the rest of the transit to Earth vicinity

(potentially use aerocapture assist to decelerate to achieve Earth orbit)

12 - Rendezvous with the in-space assembly base

13 - Transfer crew to Orion (or CCDev) and use Orion (or CCDev) to return crew and samples to Earth 
Those operations would result not only in the conduct of an exploration mission but also in the return of the most critical of mission assets (the interplanetary propulsion system and the habitat module) to near-Earth orbit. Returning those elements to near Earth provides the capability to reuse them for the next exploration mission.

During the summer of 2012 interactions with NASA JSC propulsion and guidance, navigation and control experts lead to refinement of the mission design to reflect the intent to use nuclear powered electric propulsion. The mission profile that has resulted includes assembly at a Nuclear Safe Orbit (a minimum of $500 \mathrm{~km}$ altitude) for the ISP. The use of nuclear powered electric propulsion results in the vehicle accelerating slowly when departing from Earth (or from Mars) and when entering Mars orbit and returning to Earth orbit. Slow acceleration results in a spiral path near Earth and near Mars. To minimize crew time, the current mission has the SHM vehicle accelerating thru most of the Earth departure spiral before the crew is launched on an Orion vehicle to catch and ingress the SHM before it departs from the vicinity of Earth. This approach also addresses getting the crew thru the Earth's radiation belts quickly. An Orion is also used during the SHM return to the vicinity of Earth to enable the crew to depart the SHM and return to Earth while the SHM continues its spiral to return to the NSO assembly orbit.

Aerocapture at Earth of the SHM is not acceptable due to the nuclear power plant and the minimum $500 \mathrm{~km}$ altitude constraint. However, aerocapture is acceptable at Mars and is planned to reduce the SHM velocity to enter an elliptical orbit around Mars. The aerocapture/heat shield would also be used to provide most of the deceleration of the habitat elements to land on Mars. At the end of the habitat aerocapture maneuver, the aerocapture/heat shield would be detached and the habitat elements would propulsively land using a Habitat Propulsion System (HPS). After surface exploration, the habitat would use the HPS again to propulsively ascend to rendezvous with the ISP.

This nuclear powered electric propulsion approach results in a Mars mission that takes an estimated 585 crew days (813 total mission days) to complete. Estimates for the habitat module mass have been recalculated based on the consumables required for this length of exploration mission.

A logical progression of missions using the SHM concept would be to develop the SHM habitat and ISP (designed to conduct the most challenging Mars surface mission), conduct a near-Earth asteroid (NEA) rendezvous mission, refurbish and resupply the ISP and SHM at a near-Earth site, conduct a lunar mission to test the capabilities for a Mars mission, refurbish and resupply the ISP and SHM again at the near-Earth site, then conduct a Mars mission.

\section{B. Background}

The Presidential Budget proposal for fiscal year $2011^{1}$ contained several concepts that (if realized) can be used to improve the way deep space missions are conducted. It also directed NASA to focus on deep space missions including a mission to a NEA and ultimately to conduct a human mission to Mars.

The SHM concept of a different approach to crew accommodations and mission conduct puts the new concepts together in a way that can make exploration more efficient and less costly, and would nearly eliminate the waste of mission resources. SHM combines the following concepts: CCDev (or Orion) access of crew to LEO; heavy-lift launch vehicle (HLV) launch of large payloads (now funded as the Space Launch System (SLS) Program); fueling (later refueling) (and assembly) in space; Closed Loop Life Support (CLLS); interplanetary space propulsion (probably nuclear powered); green technology (reuse mission assets and likely use of liquid methane as the SHM propellant); possible aerocapture; and ISS utilization. As of 2012, NASA is pursuing all of those new technology and vehicle efforts as currently funded projects or in future plans. The CCDev, Orion, and SLS projects are well under way.

The SHM concept was first communicated to the NASA Human Exploration Framework Team via an email in June 2010. It was proposed as a game-changing concept to the NASA Office of the Chief Technologist in September $2010 .^{2}$ It was presented at the NASA Johnson Space Center (JSC) Innovation Day in May 2011 and at a Knowledge Capture forum in June 2011 at JSC. During those presentations, the concept was referred to as the Single Crew Module (SCM) concept. (The change to SHM was made to eliminate confusion with mission concepts others have proposed involving a single crewmember.). An earlier version of the SHM concept was presented at the 2012 International Conference on Environmental Systems ${ }^{3}$.

\section{I. Single Habitat Module Essentials}

To achieve the goal of the SHM concept to efficiently conduct an exploration mission and enable reuse of the critical elements of an exploration mission, the elements to be reused must be designed to meet the needs of the most difficult exploration mission envisioned. The most difficult mission currently envisioned is the surface exploration of Mars. Transit times for NEA and Mars missions will be many months. Exploration periods on Mars will also be 
many months. The vehicle that addresses the Mars surface exploration mission would be capable of missions to NEAs or the moon and would thus be reusable for those types of exploration missions.

The design driving case includes a Mars landing and surface exploration followed by return to Earth. Mars surface exploration requires that the habitat be equipped with propulsion capability to descend from low-Mars orbit (LMO) to land at a designated exploration site, support crew operations while on Mars, then ascend back to LMO to rendezvous with the ISP.

For the SHM approach, it is critical to return the habitat module and the Multi-Mission Space Exploration Vehicle (MMSEV) (for either in-space and surface mobility) to destination orbit then back to Earth since both the module and the MMSEV are essential for crew support and/or to address potential contingencies.

The habitat module of the SHM concept will need to address all life support and crew habitability and command functions for each entire exploration mission. It must provide enough resources for the crew to function during the long zero gravity (or very low acceleration of the ISP) of the to-and-from transit phases and also be compatible with the long-duration surface exploration phase in the partial gravity of Mars or the moon.

Cabin atmosphere leak or contamination and other contingencies must be addressed.

Extravehicular activity (EVA) and mobility capabilities are required to enable exploration at the destination.

\section{I I . Single Habitat Module Concept Implementation}

If implemented, the SHM concept will have a program to develop the best design for the variety of elements. Design development of the SHM would consider many aspects of the mission to use the technology available during the design period to accomplish the SHM goals. The Constellation (Cx) Program and technology development efforts have provided many options for how to implement such a concept. Many options are possible for the technologies involved in specific parts of each deep space mission. The new Advanced Exploration Systems Program and Office of Chief Technologist efforts will verify that new technology candidates are ready to make the new vehicles robust and efficient in accomplishing mission goals. Mass minimization and safety will be key design considerations. Considering technology options that are or are expected to be available and vehicle element concepts, the features described in the following section and in Fig. 2 make sense for potential design solutions for the SHM concept.

The CLLS, thermal control, EVA, habitation, and command and habitation capabilities (needed to support the crew during the entire mission) need to be as efficient as possible to minimize mass of both equipment and consumables. Long-duration missions have been shown in exploration trade studies to greatly benefit from regenerating resources. The longer the mission, the more beneficial regeneration of resources becomes. A single habitat and command module will take advantage of the benefits of regeneration for the entire duration of the mission. The most reliable solution will be employed, combined with appropriate redundancy and sparing.

The CLLS will minimize waste products, which will partially address planetary protection issues. The CLLS implemented will have to address the most demanding of the environments, whether that occurs in transit or during surface operations.

Thermal control radiators must address the peak heat loads in transit and at Mars. Radiators that work efficiently in deep space may also work well in a convective Martian atmosphere.

EVA capabilities will be required at the exploration destination. EVA interfaces would need to be robust to address the dust environment and provide the isolation needed for the crew from potential Martian contamination. The suit port concept ${ }^{4}$ would provide very efficient EVA capability and would address the dusty environment. The same EVA system can address potential contingencies during transits and at the destination.

Including an MMSEV would address the exploration mobility and EVA capability needed at the destination. The MMSEV would also be very capable of addressing many potential cabin contingencies since it can function for an extended period of time as an independent spacecraft and offers independent EVA capabilities.

Landing gear is required for the lunar and Mars missions. The landing gear could be detached and left on the surface to reduce return mass. 


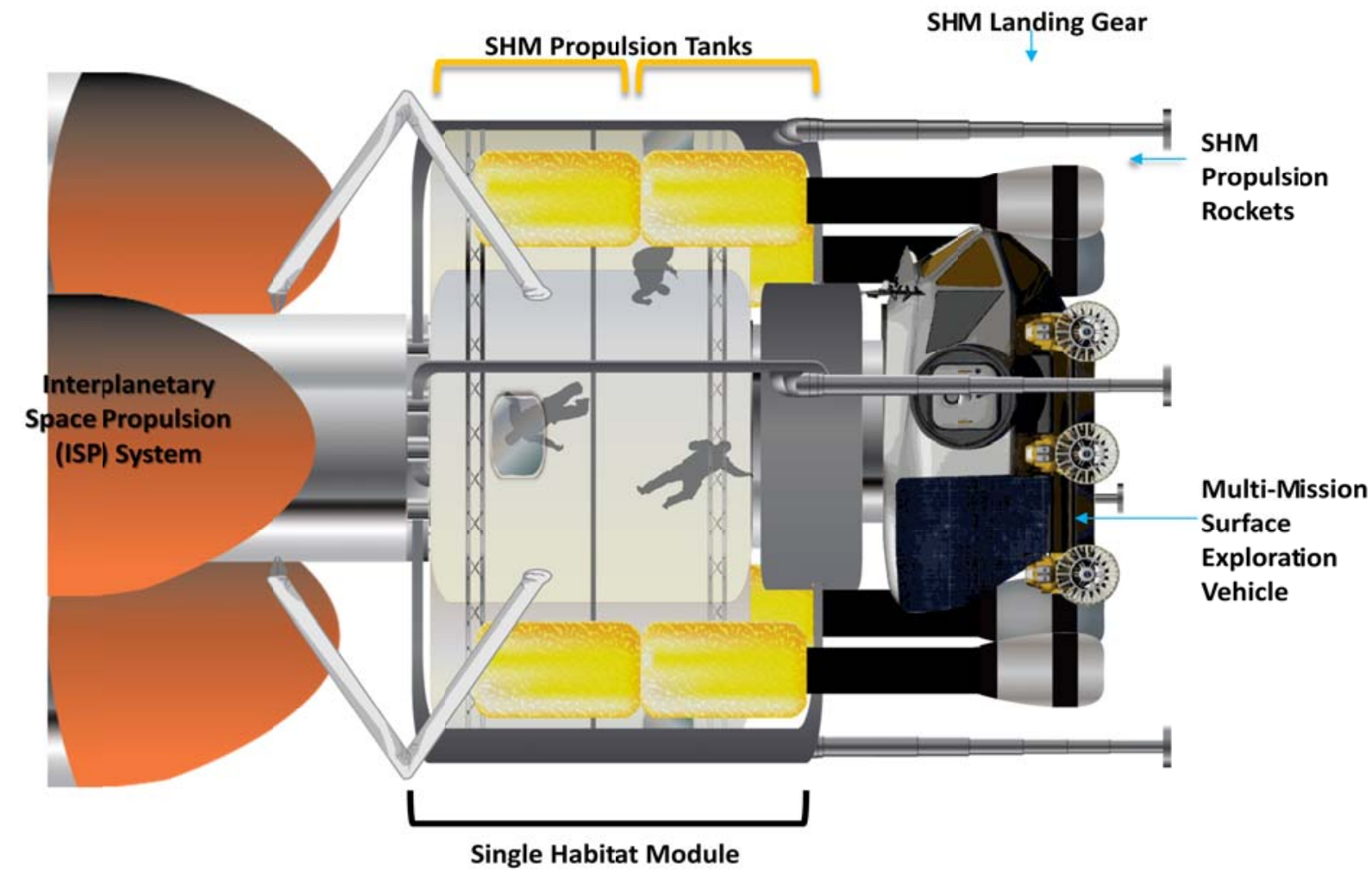

Figure 2. SHM propulsion, MMSEV, and ISP provide radiation protection for the habitat.

\section{A. Habitat Propulsion System Implementation}

Prior Cx exploration scenarios to the moon ${ }^{5}$ or Mars $^{6}$ required a propulsion system that had to deliver a longduration habitat for crew occupation to the surface. The SHM concept requires that the habitat be returned to orbit to rendezvous with the ISP. The propulsion system of planned Cx missions (which has been assessed to be feasible) had significant capability. The primary difference between the Cx and SHM concepts is in the amount of fuel the habitat propulsion system requires to perform the ascent. Considering that the landing gear is probably left on the surface, and given enough propellant, it seems that a system capable of launching the habitat from the $.377 \mathrm{~g}$ Mars gravity well should be feasible.

Cx concepts had not addressed the need to have redundancy in the descent and ascent propulsion systems or long-term radiation exposure for a deep space mission (deemed forward work when the Cx program was canceled). If the SHM propulsion system is provided in four modular rockets attached to the habitat in space (as shown in Fig. 2), the system could provide the capability to address a one-engine failure contingency during descent or ascent at the destination.

Liquid methane/liquid oxygen (MOX) propulsion is probably the best candidate for long-duration exploration missions since long-term thermal storage of those propellants is feasible (long-term storage of liquid hydrogen is problematic due to the extremely low (around 20 Kelvin $(\mathrm{K})\left(-253^{\circ} \mathrm{C}\left(-423^{\circ} \mathrm{F}\right)\right)$ temperature required to store liquid hydrogen). Thermal conditioning via deep space cooling ${ }^{7}$ can address the liquid methane (approximately $111 \mathrm{~K}$ ($\left.162^{\circ} \mathrm{C}\left(-259^{\circ} \mathrm{F}\right)\right)$ and liquid oxygen $\left(90 \mathrm{~K}\left(-183^{\circ} \mathrm{C}\left(-297^{\circ} \mathrm{F}\right)\right)\right)$ thermal storage requirements. Cryo-coolers may be required to address storage thermal conditioning while at either the moon or Mars.

Summer 2012 trajectory assessments have confirmed that aerocapture makes sense to enter Mars orbit then to provide braking prior to landing. An aerocapture/heat shield will be used below the MMSEV to aid in the deceleration of the entire SHM to enter Mars orbit. The same aerocapture/heat shield is then used after habitat separation to decelerate the habitat during Mars descent. The aerocapture/heat shield is to be detached prior to landing to enable habitat landing. The habitat propulsion system is required to complete the maneuvers to land on Mars then to launch the habitat to rendezvous with the ISP in Mars orbit. 


\section{B. Addressing Radiation Protection}

Propellant tanks with MOX surrounding the habitat would provide a significant, high-quality radiation barrier while fueled. It is likely that the SHM propulsion system would be detached from the SHM after rendezvous with the ISP to minimize mass of the return vehicle back to Earth.

Positioning the MMSEV at the front end of the SHM provides radiation protection while in transit both to and from the destination. It also addresses access to the destination surface since it will be near to the surface after landing. Lunar surface systems studies identified a significant problem associated with getting mission elements from the top of the descent stage to the surface. The SHM mounting of the MMSEV between landing gear effectively addresses that issue.

The ISP provides a radiation barrier at the aft end of the habitat.

\section{Interplanetary Space Propulsion System Implementation}

ISP system efficiency is critical to the feasibility of deep space exploration. Chemical propulsion can work, but the mass required would be very high. If electric propulsion technology development efforts are successful, that technology promises to provide a dramatic improvement in propulsion efficiency. Due to the dramatic specific impulse achievable via electric propulsion, a factor of 10 less propellant might be required (versus Space Shuttle vintage chemical propulsion).

High power is required to achieve both the high specific impulse and moderately high thrust desired for deep space transit. To provide the high power levels needed probably requires that nuclear (versus solar electric) power be employed. If thrust levels are high enough, these dramatic changes in mission planning are achievable:

1) Gravity-assisted processes might become feasible during transit (e.g., separation of gas and liquids) (such processes might also be compatible with the partial gravity of the moon or Mars).

2) The effect of low or zero gravity on bone density and muscle mass and strength would be lessened. The crew would be stronger at their destination and thus better able to conduct exploration.

3) The time required to reach destinations will be less, and different trajectories can be considered. (Mission constraints related to Hohmann transfer trajectories may not be as significant to mission planning.)

4) The length of time the crews are exposed to space radiation is reduced, thus alleviating concerns of longduration radiation exposure.

\section{Near-Earth Assembly of Single Habitat Module Mission Elements}

The SHM concept assumes that the capabilities developed during the assembly and operation of the International Space Station can be used to address assembly of exploration vehicles. Thus, the focus of the SHM concept is on the vehicle that leaves the vicinity of Earth, conducts exploration missions, and returns to the vicinity of Earth.

The ISS was assembled during the period between 1998 and 2010, and it demonstrated the capability to assemble large integrated structures in space. That capability could be employed to create a base to assemble and fuel SHM modules. It is envisioned that a U.S. photovoltaic module linked with a U.S. laboratory module with Canadian robotic capabilities and probably a Russian Service Module would provide the capability to assemble the SHM. Such an assembly base would be placed in a location near Earth that maximizes the payload delivery capability of the Space Launch System. The SHM modules would be delivered and assembled and then fueled at that base to enable starting exploration missions. The use of nuclear powered propulsion requires that assembly of the vehicle involving the ISP be at or above a Nuclear Safe Orbit of $500 \mathrm{~km}$ latitude.

Once the SHM is fully outfitted and fueled the mission would start with the SHM accelerating in a spiral away from Earth. The exploration crew is to be launched via Orion (or CCDEV) to ingress the SHM near the end of the spiral away from Earth to begin the exploration mission.

Assembling the SHM in space relaxes launch vehicle-related architecture constraints since the launch vehicle shroud is a restriction only for each launch. This flexibility may become important when the overall design of the SHM vehicle is addressed since the diameter of launch vehicle shrouds is a significant constraint.

During the long-duration exploration missions, the assembly base capabilities could be used for other purposes. The facility could be used for commercial or government (e.g., the development of power satellites).

It is feasible that the ISS could be employed as the location at which exploration vehicles are assembled. That would provide an immediate and very capable platform from which to begin assembly. However, such a scenario would require that the ISS be repurposed, and it would require that HLV delivery of payloads be to the 57-degree inclination orbit, which reduces the mass of payload that can be delivered (versus lower-inclination orbits). Assembly processes involving the nuclear power plant will be required to be above the NSL $500 \mathrm{~km}$ altitude.

\section{E. Reuse of Mission Assets}


The SHM approach can be used to conduct missions to asteroids, the lunar or Mars surface, or other deep space exploration destinations (all using one vehicle, sequentially).

The reuse of SHM assets requires that the ISP and habitat be returned to the vicinity of Earth so that the crew can be transported back to Earth via CCDev or Orion, and so that those mission assets can be refurbished, refueled, and reused. Returning to the vicinity of Earth requires that the ISP decelerate the vehicle to return to the assembly base.

Aerocapture on return to Earth Orbit is attractive but is not acceptable when a nuclear power plant is part of the returning vehicle. The crew can however depart the SHM complex when the vehicle approaches Earth via rendezvous with and use of an Orion vehicle. Such an approach minimizes crew time in space by allowing the crew to return to Earth while the SHM completes its spiral entry to Earth orbit. At the end of an SHM mission, the ISP system (nearly empty of fuel) and habitat are at the orbiting assembly base. The SHM would include the habitat and the MMSEV, thus both of those modules would be available for reuse. Those core elements could be reused for subsequent exploration missions. After a lunar or Mars surface mission, the SHM landing gear would probably be left on the surface and would thus need to be replaced. The habitat propulsion system propellant tanks would be replaced as units, probably including the rockets for each of the four, to perform the next surface mission.

To reuse the SHM, a fueled set of HPS rockets would be provided as part of the refurbishment and refueling process to enable the next exploration mission. During the course of mission operations, the accumulation and processing of waste products may address radiation protection for the return-to-Earth part of the mission (to compensate for the habitat propellant that has been exhausted).

A challenge for the SHM is the durability of the habitat module and the ISP. It is not clear how formidable that challenge will be. However, each element in the Cx must operate for long periods of time; therefore, the added duration associated with subsequent flights is a challenge, but it is probably achievable.

The cleanup of the SHM and the refurbishment of equipment at the assembly station could address life limit concerns. Cleanup and refurbishment and refueling operations could be contracted to commercial organizations.

The SHM concept would be used for exploration but not for colonization since no base infrastructure would be left behind. Once exploration has established targets for colonization or conduct of business, the Cx Lunar Surface Systems (LSS) concepts should be used to meet colonization and/or commercialization needs.

\section{F. Why Single Habitat Module is Expected to Reduce Cost and Time Required to Explore}

The SHM concept should save a significant amount of mass-to-destination when the entire mission is considered since prepositioning a habitat and separate descent and ascent vehicles is not required. Fewer elements of exploration missions should result in streamlined, focused development. Development of fewer vehicles should reduce the schedule to start exploration. Efficiencies in development organizations needed and the cost to conduct missions will be realized.

Reusing mission assets for subsequent missions will dramatically reduce the cost and schedule for other exploration missions.

The in-space assembly base (perhaps ISS, but more likely ISS-derived) could be used to support Earth orbit and other Earth vicinity (Lagrangian or lunar) NASA or commercial activities between exploration missions.

\section{An Approach for Evaluating the Single Habitat Module Concept}

Based on the SHM concept essentials and a potential implementation of the concept, a top-level evaluation of the concept can be performed to determine the merits of this concept versus other exploration approaches. Only qualitative assessments can be made at this stage of the concept since details of the concept implementation have not been established. The top-level overall mission benefits of the SHM concept versus separate transit, descent and ascent, and surface habitats can be assessed.

Evaluation of the SHM concept can start with a mass estimate of the habitat needed for the transit and the exploration phases of a Mars surface mission. Using the habitat mass estimate, the habitat propulsion system capability and mass can be calculated. Once an estimate of the mass of those elements is calculated, the capabilities and mass of the ISP system can be calculated. Combining the habitat, habitat propulsion system, and ISP masses results in an estimate for the total SHM vehicle for use in space exploration.

Evaluation of the SHM concept can alternatively focus on the major elements of the SHM concept versus those of other exploration concepts. Such a comparison can establish the fundamental differences in approaches.

\section{A. Mass Estimates for the Single Habitat Module Concept}

To establish the feasibility of the SHM concept, an estimate of the mass of the crew habitat needed for longduration missions was combined with the mass estimates for an MMSEV and landing gear mass from Cx Altair to 
estimate the habitat mass. Much of that information can be derived from Cx LSS or from more recent Human Architecture Team (HAT) studies. An estimate for the habitat from HAT studies of the May 2011 Deep Space Habitat is relevant.

Interaction with propulsion and trajectory experts has resulted in refinement of the approach for calculating the SHM mission mass. Assuming that nuclear powered electric, high specific impulse propulsion is used and a minimum energy transfer to Mars results in constraining the mission start date but results in a calculated mission transit time. Assuming a feasible 5 megawatt nuclear power plant and electric variable specific impulse engine, the transit time can be calculated. Using an aerocapture approach for deceleration of the SHM at Mars and to partially decelerate the habitat during landing; the mass of aerocapture/heat shield and the velocity change the Habitat Propulsion System (HPS) is required to provide can be calculated. Assuming a surface stay time that is adequate to conduct exploration allows calculation of the mission length (when combined with an assumption that the SHM decelerates to return to Earth orbit). Assuming that the crew egresses the SHM near Earth at the start of the deceleration spiral at Earth allows one to complete the calculation of the time the crew spends to conduct the mission.

Using the total time of the mission and the time the crew is onboard allows calculation of the consumables required for the mission. Knowing the consumables mass allows the habitat mass to be recalculated. Using the habitat mass, the HPS mass and performance can be calculated. Then the combined habitat and HPS mass can be used to recalculate the trajectory and converge on a solution for the total mass of the SHM concept.

The first iteration of the SHM mass has been calculated and a mission trajectory has been predicted. A mission length of 585 days while the crew is onboard (assuming a 30 day surface exploration) was calculated. The SHM will accelerate away from Earth in a spiral for 166 days prior to crew arrival. The crew will egress the SHM and return to Earth (probably via an Orion vehicle) at the start of the spiral of the SHM to return to the NSO around Earth. The remaining spiral will take around 66 days for the uncrewed SHM. So the total transit time for the SHM is 813 days and the crewed part of that time is 585 days.

The trajectory analysis and first iteration mission mass estimates are shown in the summary below. This data is prior to calculation of the mass of the HPS - the HPS mass is expected to be significant.

\section{Trajectory Results Summary}

- IMLEO $=249 \mathrm{mt}$

- Does not include habitat descent, ascent, or rendezvous propellant

- Earth escape spiral

- Assumed 7000sec Isp

- Propellant mass $=25 \mathrm{mt}$

- Time $=165$ days

- Earth-Mars heliocentric transfer

- Departs 12/12/2028

- Variable Isp

- Propellant mass $=36 \mathrm{mt}$

- Time $=248$ days

- 30 Mars surface stay
- Mars escape spiral

- Assumed 3000sec Isp

- Propellant mass $=15 \mathrm{mt}$

- Time $=18$ days

- Mars-Earth heliocentric transfer

- Departs 10/7/2029

- Variable Isp

- Propellant mass $=43 \mathrm{mt}$

- Time $=283$ days

- Earth capture spiral

- Assumed 7000sec Isp

- Propellant mass $=10 \mathrm{mt}$

- Time $=66$ days

\section{Total Crew Time $=\mathbf{5 8 5}$ days Total Vehicle Time $=813$ days}


The mission length has been used to calculate the mass of the habitat consumables. Recent data for the design of MMSEVs has been used to update the mass of that part of the habitat module. An estimate of $20 \%$ of the mass of the SHM complex (as it arrives at Mars) $(27,000 \mathrm{~kg}$ ) for the aerocapture/heat shield has been used.

The calculation of the habitat propulsion system mass is in work. Once that calculation is complete a second iteration trajectory analysis will be performed to refine the total SHM Mars mission mass.

a. Total habitat mass for a four-crew 585-day mission is $31,000 \mathrm{~kg}(68,200 \mathrm{lb})$

b. Habitat landing system - estimate (2000 kg (4409 lb)) based on Cx Altair estimates

c. MMSEV would be based on lunar estimates - approximately $6547 \mathrm{~kg}(14,400 \mathrm{lb})$

Those elements result in an estimate mass of habitat, landing system, MMSEV of around 39,600 kg (87,120 lb).

The HPS will provide the capability to safely land that equipment then return a subset to LMO. The mass to be returned to LMO would be the habitat plus SEV or $37,600 \mathrm{~kg}(70,548 \mathrm{lb})$ since the landing gear could be left on the surface.

An estimate of the total mass of the SHM requires a calculation of the propulsion system and the required propellant (the HPS) mass. That estimate is future work at this time. However, based on the logic presented above, the propellant system seems feasible since the added mass (versus Cx concepts) is primarily the mass of the propellant needed by the habitat propulsion system to return to LMO.

The combined habitat and HPS vehicle mass can be used with estimates for the high specific impulse ISP systems to estimate the thrust needed for the ISP and the amount of fuel the ISP would need to deliver the SHM to and from LMO.

\section{B. Comparison of Single Habitat Module Elements to Those of Apollo and Constellation Exploration Plans}

The SHM concept is not directly comparable to past exploration approaches since it employs technology advances that were not considered in the past, and since it reuses mission assets. The SHM approach of developing an exploration capability that can be employed for any exploration destination results in a significantly different compliment of mission assets than other approaches.

SHM could be compared directly to the Cx Mars Design Reference Mission (DRM) to compare assets and thus understand the benefits. Alternatively, SHM could be compared to the elements required to conduct a lunar sortie mission. Those comparisons would address the benefits of the mission simplification associated with the SHM single habitat. However, such comparisons would be somewhat misleading since one of the benefits of the SHM concept is the reuse of mission assets and, when compared to a single mission, those benefits are not considered.

The most appropriate comparison of past approaches and SHM is to compare the combination of NEA, lunar landing, and Mars surface missions. The Cx DRMs require the following: separate crew accommodations for transit, descent, ascent, and surface operations; new vehicles for every mission; prepositioning mission assets at exploration locations; (for the Mars DRA) in-situ resource utilization to provide propellant for the return mission. The SHM simplifies the mission compliment of elements.

A top-level comparison of elements required to depart from Earth for those exploration missions finds that those elements required are:

SHM for the three missions - Habitat, MMSEV, ISP plus two refurbishment and refuel operations.

HAT NEA - Orion, DSH, MMSEV, Solar Electric Propulsion

Cx Lunar Sortie mission - Orion, Altair (Lander, Ascent)

Cx Mars Surface mission - Orion, DSH, Surface Habitat, two Nuclear Thermal Rockets (NTRs), SEV, Combined Descent and Ascent Vehicle (DMAV), Mars Ascent Vehicle (MAV).

Comparing elements required for the approaches can lead to an appreciation of the ways that the approaches are different. Qualifications to such comparisons are that the masses of the SHM habitat and ISP propulsion systems still need to be refined, and the SHM refurbishment and refuel process will require launch of materials from Earth. The author speculates that NEA and Cx Mars DRM missions would also require some level of assembly near Earth prior to departure. The SHM approach of decelerating the ISP and SHM to return to Earth vicinity will require more propulsion capability than the direct atmospheric reentry planned for the HAT NEA and Cx lunar sortie and Mars DRMs.

\section{Summary of Single Habitat Module Approach Benefits}

The benefits of the SHM approach center around the single vehicle needed to conduct exploration. Supporting the crew through the entire mission in one habitat simplifies the total mission and enables reuse of mission assets. 
The SHM approach can make exploration more affordable and can focus missions on exploration instead of vehicle development. SHM approach benefits include:

1) A single module that addresses crew functions for all mission phases.

a. This eliminates modules that in Cx approaches are required for transit to a destination (MPCV), transit from orbit to destination surface (Altair descent vehicle), a surface habitat, and an ascent vehicle to return to orbit.

2) Elimination of the need to develop new vehicles for subsequent missions

3) Transportation of significantly less mass to destination since fewer elements are required

4) Use of regenerative technologies minimizes mass via use for the entire mission

5) Elimination of the need for short-duration non-regenerative technologies

6) No requirement for the prepositioning of assets

a. Reduces landing accuracy requirements

b. Crew arrives at an exploration site that has not been explored robotically via prepositioned assets (thus crew exploration is all new and not partially redundant)

7) Use of a high-power ISP that can shorten mission duration from versus the Mars DRA (585 crewed days versus the Mars DRA of over 860 crewed days)

a. Would reduce the amount of consumables required

b. Would partially address radiation protection by shortening crew exposure time

8) Vehicle dimensions that are not constrained by launch vehicles

a. Allows architectural freedom to arrange mission elements

9) The positioning of propellant around the habitat protects the crew from radiation

10) Exploration flexibility by allowing the exploration community to use exploration resources to get to new destinations instead of building new vehicles

\section{Summary and Conclusion}

The SHM concept has merits and could significantly simplify the conduct of exploration missions. The reuse of mission assets for subsequent exploration missions could dramatically reduce the cost of exploration and could significantly reduce the time required to develop and conduct a Mars mission. Assessment of the use of high specific impulse propulsion has lead to a better understanding of the implications using such technology has on mission planning. The infrastructure in the Orbital Assembly Base could be used to conduct other near-Earth NASA or commercial operations between exploration missions.

It is recommended that more detailed studies be conducted to confirm that the SHM concept will provide a significantly better way to conduct space exploration. Once confirmed, exploration programs should initiate development of the assembly station (or address repurposing of ISS as the assembly station) and complete technology development to ready technologies for use in a SHM program of exploration.

\section{Forward Work}

The mass of the HPS can be calculated using current calculations of the mass of the SHM habitat, the SEV (or MMSEV), and landing gear (total of 39,600 kg (87,120 lb)). Once that calculation is complete, that vehicle mass will be used to calculate the performance required of the ISP system. The mass of the ISP fuel required can be calculated using that performance and the forecasts of the specific impulse that is likely to be achieved in advanced propulsion development.

That set of calculations will complete the estimation of the total compliment of the mission elements for a Mars surface mission. The SHM approach mission mass can then be compared to the mass of comparable HAT and prior $\mathrm{Cx}$ mission estimates. The per-mission and aggregate mission masses for several exploration missions can then be compared and the approaches traded.

Given that those trades validate the advantage of the SHM approach, the next steps include the normal development activities of evaluating technology options to minimize mass and improve functionality of mission elements. Technology and operational options that could lead to reduction of vehicle mass or reduced cost include:

1) Using an inflatable habitat structure to reduce structural mass

2) Using the ISS as an assembly site (eliminates the need for a separate near-Earth assembly base, but decreases the mass each launch can deliver to space because of the high inclination)

3) Considering advanced ECLSS technologies to save more than $1000 \mathrm{~kg}$ (2205 lb) versus ISS-derived technologies (for 1 year) 
4) Using the Orion instead of the SEV for contingency and to provide direct return of the crew (without the time needed to decelerate to Earth orbit). Provides contingency capabilities and shortens crew return time. Eliminates the roving capability at the destination.

5) Jettison landing gear at destination surface (requires refurbishing the SHM with new landing gear for the next mission)

6) Jettison SHM propulsion prop tanks and maybe the engines (requires resupply of those elements for the next mission (refueling required in any scenario)

7) Segmenting the ISP prop tanks and discarding tanks when empty (refueling required in any scenario)

8) Leaving the SEV on the surface (compromises contingency capabilities during return)

9) Developing and fueling in LEO then transporting to beyond the radiation belts of Earth before crew ingress (maximizes Earth launch mass to space, minimizes crew radiation exposure)

\section{Acknowledgments}

The author gratefully acknowledges the support and encouragement from the Crew and Thermal Systems division of NASA JSC, specifically the division chief Patricia Petete, branch managers Joe Gensler and Craig Dinsmore, and illustrator Jeannie Corte.

The author gratefully acknowledges the planning for exploration missions done by the Cx Program that provided a moderate level of detail for exploration concepts. Specifically, the Lunar Surface Systems team lead by Chris Culbert and Gary Spexarth, the Mars DRA team lead by Brent Drake, and the habitation team lead by Larry Toups.

Experts at NASA JSC in propulsion and guidance, navigation and control contributed to the concept implementation with the ideas included in this paper on using the advanced high specific impulse technology for design of a Mars mission. Ellen Braden developed much of the mission design parameters relating to the SHM concept. Chris Cerimele also contributed to mission design. Gene Grush provided insights into the habitat propulsion system needed to implement the SHM concept for a Mars mission.

\section{References}

${ }^{1}$ Office of the President of the United Stated of America, "National Space Policy of the United States of America”, June 28, 2010.

${ }^{2}$ Chambliss, J. P., “Single Habitat Module (SHM) for Exploration,” Response to NASA RFI Response to Solicitation Number: NNH10UA007-1 to the Game Changing Development Program of the NASA Office of the Chief Technologist, September 2010.

${ }^{3}$ Chambliss, J. P. “The Single Habitat Module for Exploration” The 2012 International Conference on Environmental Systems, AIAA 2012-3416, July 201

${ }^{4}$ Boyle, R. M., Mitchell, K., Allton, C and Hsing, J. "Suitport Feasibility - Development and Test of a Suitport and Space Suit for Human Pressurized Space Suit Donning Tests” Paper to the 2012 International Conference on Environmental Systems, (paper number is TDB), July 2012.

5“"Lunar Capability Concept Review (LCCR),” NASA, June 18-20, 2008.

${ }^{6}$ Drake, B. G. Editor, Mars Architecture Steering Group, "Human Exploration of Mars - Design Reference Architecture 5.0”, NASA document NASA/SP-2009-566, July 2009.

${ }^{7}$ Chambliss, J. P. , Swickrath, M and Sweterlitsch, J. J. "Potential Uses of Dep Space Cooling for Exploration Missions”, 2012 International Conference on Environmental Systems, AIAA 2012-3638, July 2012. 